\title{
Measuring the Impact of Knowledge Gained from Playing FPS and RPG Games on Gameplay Performance
}

\author{
Mona Erfani Joorabchi and Magy Seif El-Nasr \\ Simon Fraser University, Surrey, BC, Canada \\ $\{$ mea16, magy\} asfu.ca
}

\begin{abstract}
Understanding players' gameplay behaviors, performance abilities, and interests is a growing research area due to its utility in developing knowledge and theory that can enhance game design. Much previous research exists within this area, however, none of these works deeply investigated player behavior and its relation to knowledge/skills gained by playing specific genres. This topic is important, as knowledge gained from playing different genres influences players' game behaviors. Knowing the details of the skills gained and their influence on performance will allow designers to make informed decisions about their game design given knowledge about target audience's playing habits. In this paper, we address this issue. In particular, we discuss results of a study conducted with 35 college participants to explore the influence of prior gaming experience, specifically with Role Playing and First Person Shooter games, on knowledge/skills related to their ability to navigate and solve spatial puzzles in 3D games.
\end{abstract}

Keywords: Game user research, Game performance metrics, Game usability, Game users, User gaming experience, Player modeling, Video games.

\section{Introduction}

Video games have been on the market since the 1980s. As a result, there are many skilled players who learned genre conventions. Designers are now challenged with creating games for ones who have accumulated skills/knowledge and others who are either casual gamers or inexperienced [7, 10]. Many previous researches have investigated the players' gameplay experience, including their emotions [15, 4], motivations [16], play styles [22, 25], and behaviour [5]. Several models were proposed to measure players' satisfaction including GameFlow [19]. In addition, several researchers proposed a set of heuristics to evaluate the game experiences. Desuvire et al. [7] proposed Game Approachability Principles as "the level of helpfulness in a computer game for new and inexperienced players." Lemay et al. [16] proposed a semantic differential approach to understand the players' various experiences regarding their attitudes by using a list of "bipolar adjectives". Authors argued that players' perception of a game/genre will affect their performance and skills. Other studies reported that mastering game tasks and challenges is connected to game enjoyment [14]. Sherry [18] stated that too easy games result in boredom, and too hard games can be fru- 
strating. On the other hand, Klimmt et al. study [14] showed that game enjoyment can change with increasing playing time. High number of successes and few failures at the beginning of a new game could increase the enjoyment however that could result in boredom when game experience improved. There are several methods for gathering players' feedback e.g., usability methods, surveys, observation, playtesting, and ethnographic methods $[6,12,13]$. Lately, game telemetry and visualization have been proposed where it provides a large quantitative, time-stamped data for tracking and analyzing user behavior. Microsoft Game Labs did extensive user testing for Halo 3 [20]. Mellon [23] used telemetry to locate gold farmers. Ducheneaut et al. [9] used logs of player chat in Star Wars Galaxies to infer patterns in social behavior. In addition, several researchers have used data from players' behavior to construct player models [3]. Bartle developed one of the first player models by studying people playing MUDs (Multi-User Dungeons) [2]. He categorized players as Socializers, Killers, Adventurers, and Achievers. Recently other researches discussed different approaches (game metrics [8], neural network [5], game AI for creating challenging opponents [11], etc.) to construct models of the players. However, none of these previous works investigated the knowledge gained from experience with particular genres and its impact on gameplay. In this paper we present a study with 35 participants to investigate the influence of experience accumulated through playing specific game genres (described by Rollings and Adams [1]), First Person Shooter (FPS) games and Role Playing Games (RPG). We believe skills gained from playing different kinds of games are varied since their game mechanics are different e.g., FPS games require good eye/hand coordination and quick reaction time [18], while RPGs are related to logical thinking and problem solving [18]. We investigate the effect of these learned skills on solving 3D spatial puzzles in 30-minutes of playing Tomb Raider: Underworld (Eidos Interactive, 2008).

\section{Pilot Study}

Our questionnaire guides us in clustering players based on their prior experience with FPS and RPG games. We sent it to 4 experts in game user research industry to ensure content face validity. We then conducted a pilot with 3 RPG experts, 3 FPS experts and 4 non-gamers. Since self report is an unreliable measure as people often underestimate the time they spend [22], the questionnaire [23] tries to remedy that by asking specific details on games based on advice from experts. The questionnaire was promising in differentiating between FPS and RPG gamers. It found RPG gamers were playing FPS games more than FPS gamers playing RPG games. Additionally, RPG gamers were more flexible in playing games in the other genre (e.g., FPS).

\section{Study Design}

35 participants were recruited from SFU SIAT. We ended up with 13 FPS, 9 RPG, 9 non-gamers, and 4 outliers who either played the game before, or were not rated as FPS/RPG/non-gamer based on the questionnaire. We did not consider outliers in the 
statistical analysis to keep the consistency. We were not interested in gender or age influences, and used homogeneous participants; They aged 21-25 (4 between 27-32) and over $80 \%$ were male. Tomb Raider was chosen because it is a 3D game (for measuring 3D spatial puzzles and navigation), fairly new (to decrease number of outliers), not RPG or FPS but had some elements of both, and a linear game with distinguishable obstacles for defining metrics (Table 1). Since most of the metrics are not subjective, one experimenter collected them manually through video coding.

Table 1. Performance metrics for measuring skill level.

\begin{tabular}{|l|l|l|l|}
\hline 1 & Total time (min) & 7 & Items collected e.g. health/diamond (yes/no) \\
\hline 2 & Number of solved obstacles & 8 & Skip the cinematic between levels (yes/no) \\
\hline 3 & Number of deaths (fall off, enemies) & 9 & Number of events of shooting at enemies \\
\hline 4 & Read instructions on screen (yes/no) & 10 & Movement (fast, medium, slow) \\
\hline 5 & Number of checking tutorials/maps & 11 & Frustration with controller (low, medium, high) \\
\hline 6 & Ask for help/hint (yes/no) & 12 & Time to solve obstacle (sec): Obstacle 1-15 \\
\hline
\end{tabular}

\section{Results}

Questionnaire: In this experiment, the dependent variables were number of RPG games played, maximum playtime per RPG game, number of FPS games played, and maximum playtime per FPS game (each participant inserted the total number of hours played per each FPS/RPG game in the questionnaire; for each participant the greatest number was considered as the maximum playtime per game). The independent variable was the prior gaming experience groups (FPS/RPG/ non-gamer). Since the significant difference between non-gamers with other two groups was seen in ANOVA, we present statistical results of FPS and RPG gamers through the Mann-Whitney U Test (Table 2). Comparing the average number of FPS and RPG games played shows RPG players played significantly more RPG games than FPS shooters, however they did not differ in the number of FPS games they have played. Comparing the average of maximum playtime per FPS and RPG games, a significant time investment difference was found between FPS and RPG gamers for playing an RPG game (RPG gamers played more, $\mathrm{p}<.05)$ but not for playing an FPS game $(\mathrm{p}>.05)$.

Game Metrics: In this experiment, the dependent variables were the numerical variables from the game metrics (Table 1). The independent variable was the prior gaming experience (FPS/RPG/non-gamer). The data was analyzed using one-Factor ANOVA (Table 3). There was a significant difference for the number of solved obstacles between FPS gamers and the other two groups, $\mathrm{F}(2,28)=8.53, \mathrm{p}<.05$. Posthoc test (Tukey) confirmed FPS players were able to solve more obstacles than RPG players and as expected non-gamers while there was no significant difference between RPG players and non-gamers. Comparing the average time (sec) took players to solve each obstacle we found that FPS gamers spent less time than RPG gamers in solving obstacles (Figure 1). ANOVA (Table 4) showed there was significant difference between non-gamers and other two groups for obstacles 1,4 , and $5(\mathrm{p}<.05)$. Results of Mann-Whitney U Test for FPS and RPG showed there was a significant 
difference in the mean time of FPS and RPG players on obstacle 4, $\mathrm{z}=-1.905, \mathrm{p}<.05$ and obstacle $11, \mathrm{z}=-2.155, \mathrm{p}<.05$.

Table 2. Mann-Whitney U Test on the grouping variables Game Genre

\begin{tabular}{|l|c|c|c|}
\hline & Man-Whitney U & $\mathbf{Z}$ & Asymp. Sig. (2-tailed)* \\
\hline No. of RPG games played & 15.0 & -2.916 & $\mathbf{. 0 0 4}$ \\
\hline No. of FPS games played & 41.0 & -1.180 & .238 \\
\hline Max playtime per RPG game & 15.5 & -2.891 & $\mathbf{. 0 0 4}$ \\
\hline Max playtime per FPS game & 49.0 & -.638 & .523 \\
\hline
\end{tabular}

$* \mathbf{p}<.05$. Significant effects are typeset in bold.

Table 3. and Table 4. ANOVA for the independent variable prior gaming experience

\begin{tabular}{|lcccc|}
\hline Dependent Variable & $\mathbf{F}(\mathbf{2 , 2 8})$ & $\mathbf{p}^{*}$ & $\mathbf{\eta 2}$ & power \\
\hline No. of solved obstacles & 8.535 & $\mathbf{. 0 0 1}$ & .379 & .948 \\
\hline No. of checking maps/tutorials & 4.024 & $\mathbf{. 0 2 9}$ & .223 & .670 \\
\hline No. of deaths & 1.592 & .221 & .102 & .308 \\
\hline No. of shooting at enemies & 1.119 & .341 & .074 & .227 \\
\hline
\end{tabular}

\begin{tabular}{|lcccc|}
\hline Dependent Variable & $\mathbf{F}(\mathbf{2 , 2 8})$ & $\mathbf{p}^{*}$ & $\mathbf{\eta 2}$ & power \\
\hline Obstacle1 & 4.317 & $\mathbf{. 0 2 3}$ & .236 & .703 \\
\hline Obstacle4 & 4.539 & $\mathbf{. 0 2}$ & .245 & .726 \\
\hline Obstacle5 & 5.434 & $\mathbf{. 0 1}$ & .287 & .803 \\
\hline
\end{tabular}

Analyzing qualitatively we noticed Obstacle 1 and 2 included a flaming hallway, a switch to open a door and climbing on a ledge. Non-gamers spent more time getting used to the controllers, the game environment and the abilities while FPS/RPG gamers just passed (majority of players did not notice the ledge right away). Obstacle 3 involved new game mechanics for all the groups. Players need to get close to the edge of the pit to jump and grab the narrow ledge on the wall to pass the pit. Many participants reported the ledges were unnoticeable which explains the peak for obstacle 3 in Figure 1. This was evident especially with, $23 \%$ of FPS, $25 \%$ of RPG and $67 \%$ of non-gamers asked for help from the observer after spending a good amount of time. The observer believed that FPS gamers being faster and their attitude tended to be more risky and impatient e.g., FPS gamers jumped toward the wall or the pit while they still did not find the ledges. The mechanics used for obstacles 4-5 are similar to obstacle 3. FPS and RPG gamers did not differ much, but compared to non-gamers there was a big difference. This is because FPS and RPG gamers are used to learn from games and use the mechanics again. Thus their learning time can be much quicker than non-gamers. Obstacle 6 requires players to dive underwater where they may encounter sharks. They can either escape or shoot at sharks; $85 \%$ of FPS, $66 \%$ of RPG and $56 \%$ of non-gamers shot at the attacker sharks. This is due to FPS gamers are used to shooting within the games they play. Obstacles 7-9 needed players to navigate underwater to find two keys. Players' behaviors were almost the same for those 
who could get through (eight FPS, three RPG and two non-gamers). The majority of players got lost in obstacles 7-8, where they believed game design could be improved through using more efficient lighting and maps. Obstacles 11-12 were a combination of jumping and climbing in a dark room. FPS gamers generally spent less time and were able to navigate with little problem. Although some of the FPS gamers claimed they were PC gamers, the differences captured through qualitative observations among FPS gamers were minimal. For RPG gamers the difference between PC gamers and those who are used to controllers was more pronounced. Non-players showed the highest frustration level and the slowest movement, whereas FPS gamers showed the lowest frustration level and the fastest movement. Additionally, analysis showed there was a significant strong positive correlation between the number of FPS games played and the number of solved obstacles $r=.548(31)$, $\mathrm{p}$ (two-tailed)<.01 but not with number of RPG games played. There was a significant strong positive correlation between hours spent weekly on playing video games and the number of solved obstacles $\mathrm{r}=.443(31)$, $\mathrm{p}$ (two-tailed)<.05.

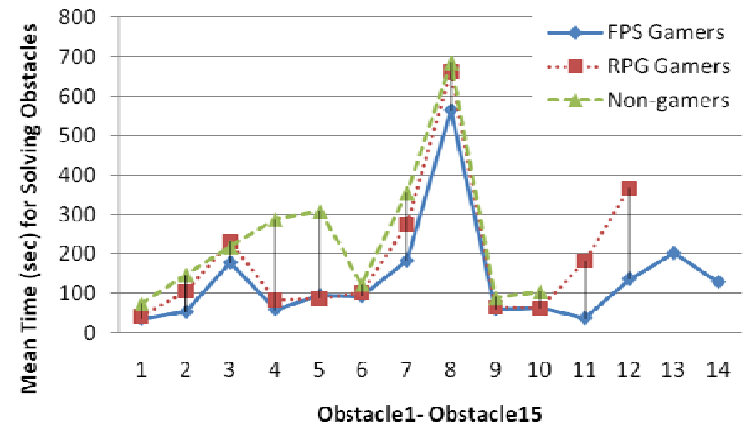

Fig. 1. Average time (sec) for solving Obstacle1-15

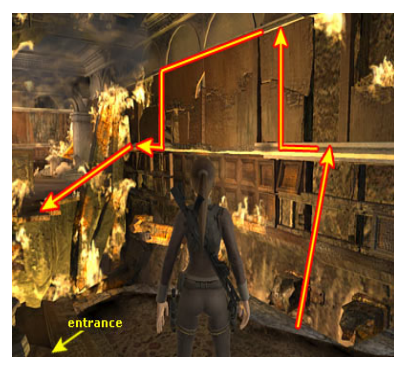

Fig. 2. Obstacle $3[21]$

\section{Conclusions}

The results implied that non-gamers' behavior was more similar to RPG gamers than FPS gamers in terms of the time it took them to solve the puzzles; there were five obstacles with significant time difference between FPS and non-gamers (Obstacles 1, 2, 4-6) while there was only one obstacle that yielded significant differences between RPG and non-gamers (Obstacle 5). We believe visual search and/or the actual learning abilities cause these differences. Another interesting finding was that the more FPS games they played the more obstacles they solved; this was not true for RPG games. By uncovering these skills further, we can develop theories that can guide developers into designing more accessible games. It should be noted that this is a difficult research area and although we tried to keep the limitations of the study low, there are some. Having only one rater for observation of frustration and movement may not be enough to establish validity and more participants is needed to establish 
generality. However, the amount of time for video coding outweighed this desire. Further research is needed to investigate what abilities give FPS gamers the observed playing navigation difference, is it visual attention, visual search or reaction time difference? In addition, this study only looked at RPG and FPS gamers, what other differences exist in terms of skills in 3D games between different types of players?

Acknowledgments. Our thanks to Dan Taylor, Tim Nichols, Bill Fulton, and Janus Rau for their knowledge on the questionnaire and Dr. Bernhard Riecke.

\section{References}

1. Adams, E., Rollings, A.: Fundamentals of Game Design. Prentice Hall, Englewood Cliffs (2006)

2. Bartle, R.: Hearts, Clubs, Diamonds, Spades: players who suit MUDs. International Journal of Virtual Reality 6, 11-16 (1996)

3. Bateman, C., Boon, R.: 21st Century Game Design. Charles River Media, California (2005)

4. Calvillo-Gámez, E.H., Cairns, P., Cox, A.L.: Assessing the Core Elements of the Gaming Experience. In: Evaluating User Experience in Games. Springer, UK (2010)

5. Charles, D., Black, M.: Dynamic player modeling: A framework for player-centric digital games. In: Proceedings of the International Conference on Computer Games: AIDE 2004 (2004)

6. Davis, J., Steury, K., Pagulayan, R.: A survey method for assessing perceptions of a game: The consumer playtest in game design. Game Studies: The International Journal of Computer Game Research 5 (2005)

7. Desurvire, H., Wiberg, C.: User Experience Design for Inexperienced Gamers: GAP Game Approachability Principles. In: Bernhaupt, R. (ed.) Evaluating User Experience in Games, ch. 8. Springer, London (2010)

8. Drachen, A., Canossa, A., Yannakakis, G.N.: Player modeling using self-organization in Tomb Raider: underworld. In: CIG 2009 (2009)

9. Ducheneaut, N., Moore, R.J., Nickell, E.: Designing for sociability in massively multiplayer games: an examination of the "third places" of SWG. In: Proc. Other Players 2004 (2004)

10. Fortugno, N.: The strange case of the casual gamer. In: Isbister, K., Schaffer, N. (eds.) Game Usability: Advice from the Experts for Advancing the Player Experience (2008)

11. Houlette, R.: Player Modeling for Adaptive Games. In: AI Game Programming Wisdom II, pp. 557-566. Charles River Media, Inc., Hingham (2004)

12. Jørgensen, A.H.: Marrying HCI/Usability and Computer Games: A Preliminary Look. In: Proceedings of NordiCHI, Tampere, Finland (2004)

13. Kim, J.H., Gunn, D.V., Schuh, E., Phillips, B.C., Pagulayan, R.J., Wixon, D.: Tracking Real-Time User Experience (TRUE): A comprehensive instrumentation solution for complex systems. In: Proceedings of CHI, Florence, Italy, pp. 443-451 (2008)

14. Klimmt, C., Blake, C., Hefner, D., Vorderer, P., Roth, C.: Player Performance, Satisfaction, and Video Game Enjoyment, Entertainment Computing. In: IFIP 2009 (2009)

15. Lazzaro, N.: Why we play games: Four keys to more emotion in player experiences. In: Proceedings of GDC (2004) 
16. Lemay, P., Lessard, M.M.: Investigating Experiences and Attitudes Towards Videogames using a Semantic Differential Methodology. In: Evaluating User Experience in Games (2010)

17. Mellon, L.: Applying Metrics Driven Development to MMO Costs and Risks. Versant Corporation (2009)

18. Sherry, J.L.: Flow and media enjoyment. Communication Theory 14, 328-347 (2004)

19. Sweetser, P., Wyeth, P.: GameFlow: a model for evaluating player enjoyment in games. Computers in Entertainment 3 (2005)

20. Thompson, C.: Halo 3: How Microsoft Labs Invented a New Science of Play. Wired Magazine 15(9) (2007)

21. Tomb Raider Underworld Walkthrough and Game Guide, http://www.tombraiderchronicles.com/underworld/walkthrough/i ndex.html

22. Williams, D., Consalvo, M., Caplan, S., Yee, N.: Looking for gender (LFG): Gender roles and behaviors among online gamers. Journal of Communication (2009)

23. Questionnaire,

http://www.sfu.ca/ mea16/instrument/questionnaire.pdf 